\title{
Persistence with statin therapy in Hungary
}

\author{
Zoltan Kiss', Laszlo Nagy ${ }^{1}$, Istvan Reiber ${ }^{2}$, György Paragh ${ }^{3}$, Mark Peter Molnar ${ }^{4}$, György Rokszin ${ }^{5}$, \\ Zsolt Abonyi-Toth ${ }^{5}$, Laszlo Mark ${ }^{6}$
}

1 MULTI GAP Study Group, Budapest, Hungary

2St. George Fejer County Hospital, $4^{\text {th }}$ Department of Medicine, Szekesfehervar, Hungary

${ }^{3}$ Medical and Health Science Centre, University of Debrecen, $1^{\text {st }}$ Department of Medicine, Debrecen, Hungary

${ }^{4}$ Corvinus University, Budapest, Hungary

${ }^{5}$ Rx Target Statistical Agency, Budapest, Hungary

6Pandy Kalman Bekes County Hospital, 2nd Department of Medicine - Cardiology,

Gyula, Hungary

Submitted: 7 January 2013

Accepted: 13 April 2013

Arch Med Sci 2013; 9, 3: 409-417

DOI: 10.5114/aoms.2013.35327

Copyright @ 2013 Termedia \& Banach

\section{Abstract}

Introduction: Persistence with lipid-lowering drug therapy by cardiovascular patients in Hungary has not been studied previously. This study was designed to determine the rate with which Hungarian patients with hyperlipidemia persist in taking lipid-lowering agents, and to compare this with rates reported from other countries.

Material and methods: This was a retrospective study that utilized data from the Institutional Database of the National Health Insurance Fund to analyze persistence rates with statins and ezetimibe. The study included data for patients who started lipid-lowering therapy between January 1, 2007, and March 31, 2009. Variables included type of lipid-lowering therapy, year of therapy start, and patient age. Main outcome measures were medians of persistence in months, percentages of patients persisting in therapy for 6 and 12 months, and KaplanMeier persistence plots.

Results: The percentage of patients who persisted with overall statin therapy was $46 \%$ after 1 month, $40.3 \%$ after 2 months, $27 \%$ after 6 months, and $20.1 \%$ after 12 months. Persistence was slightly greater for statin therapy started during 2008 than during 2007. Older patients were more persistent with therapy than younger patients. Persistence with the combination of ezetimibe-statin therapy was greater than with statin or ezetimibe monotherapy.

Conclusions: Persistence with statin therapy by patients in Hungary was low compared with other countries. Low persistence may have negated potential clinical benefits of long-term statin therapy.

Key words: persistence, adherence, statins, ezetimibe, Hungary.

\section{Introduction}

Hungarians have a higher risk of premature death due to cardiovascular disease (CVD) compared with residents of western European countries $[1,2]$ and the lowest life expectancy at birth (72.4 years) among area nations in the Organization for Economic Cooperation and Development (OECD) [3]. The importance of lipid-lowering therapy is well established $[4,5]$. Regarding the Hungarian lipid goal achievement rates in recent years there are significant improvements, but the result is still far from expec-
Corresponding author: Laszlo Mark MD, PhD $2^{\text {nd }}$ Department of Internal Medicine - Cardiology Pandy Kalman Bekes County Hospital Semmelweis u. 1 P.O. Box 46 5701 Gyula, Hungary Phone: +36-209288053 Fax: +36-66526543 E-mail: mark@pandy.hu 
tations [6-8]. In a 2007 summary of 14 key clinical trials of lipid lowering conducted since 2004 and involving 90056 individuals [9]. Ray et al. determined that statin therapy was safe and significantly reduced the cardiovascular endpoints. Furthermore, a recent meta-analysis of data from 170000 participants in 26 randomized trials of statin therapy demonstrated that more intensive lowering of lowdensity lipoprotein cholesterol (LDL-C) safely produced significant further reductions (15\%; $95 \%$ confidence interval (Cl) 11-18; $p<0.0001)$ in major vascular events, with no evidence of a threshold within the cholesterol range studies below which further reductions did not result in incremental reductions in CVD risk [10]. Even among those attaining $1.8 \mathrm{mmol} / \mathrm{l}$ or lower with a standard statin regimen, further reduction yielded benefit (relative risk (RR) 0.63, 99\% $\mathrm{Cl} 0.41-0.95 ; p=0.004$ ).

International clinical trials have shown that the optimal cardiovascular advantages of statin therapy are achieved only with the patient's long-term persistence (i.e., continuing the treatment for the prescribed duration) and adherence (i.e., extent of conformity with the prescribed timing, dosage, and frequency of a regimen) [11, 12]. The earliest point in some trials at which patients who persisted with active therapy began to experience cardiovascular advantages of statin compared with placebo therapy (the point of visual divergence - PVD) [13] was at 6 [14] or 12 months of therapy [15]. In a study that assessed patient adherence just after hospitalization, Ho et al. found that patients who discontinued statin therapy 1 month after initiation following an acute myocardial infarction had significantly lower 1-year survival compared with those who continued with statin therapy [16].

Lack of persistence with medications in general, and with statin therapy in particular, remains a widespread problem worldwide [17-19]. Several decades of research indicate that up to $20 \%$ of all patients do not fill a new prescription, regardless of diagnosis, and approximately one half of those who do fill a new prescription discontinue therapy in the first 6 months [20]. Up to $69 \%$ of all medication-related hospital admissions are due to suboptimal medication adherence [19]. Lack of persistence with statin therapy in particular has been associated with increased cardiovascular events (odds ratio (OR) 1.40, $95 \% \mathrm{Cl} 1.35-1.45$ in primary prevention; OR 1.59 , 95\% Cl 1.51-1.68 in secondary prevention) and increased all-cause mortality (hazard ratio (HR) 1.25, $95 \% \mathrm{Cl} 1.09-1.42 ; p=0.001$ ) [17, 18, 21-23]. In addition, statin therapy of insufficient persistence is associated with higher hospitalization rates and total direct healthcare costs compared with good adherence to therapy over the first 2 years of use [24].

Potential barriers to filling and adhering to prescription treatments include the presence of psy- chological disorders (e.g. depression) or cognitive impairment; adverse effects, costs (and copayment status), and complexity of medication regimens; patients' lack of insight into their illnesses and/or belief in the benefits of their treatments; and poor provider-patient relationships, such as inadequate follow-up or treatment planning and/or missed appointments; and logistical issues such as transportation problems/inconvenience/long pharmacy wait times, and/or having a sufficient supply of medications at home $[19,25]$.

Currently, only a few studies address persistence with statin therapy, the degree to which this therapy contributes to reduction of cardiovascular events, or effects of the National Health Insurance Fund payments for statin therapy on improving the health of the general population in Hungary [26].

We undertook the current study to determine persistence with statin therapy of Hungarian patients with hypercholesterolemia, and to compare this with persistence of therapy in other countries. The study examined general persistence, as well as persistence with initial therapy and persistence with therapies containing ezetimibe in certain groups. The study also evaluated the influence of statin, age, and geographic location on persistence.

\section{Material and methods}

This was a retrospective study of data maintained in the drug-dispensing database of the Hungarian National Health Insurance Fund, which contains prescription data for all patients receiving health insurance subsidies. The study included data for all patients of any age and either sex who started lipidlowering therapy with a statin, ezetimibe, or a combination of a statin and ezetimibe between January 1 , 2007, and March 31, 2009. To help ensure that only patients who began therapy during this period were included, data for any patient who received such therapy in 2006 were excluded. Data were also excluded for patients who received a combination of a statin and any lipid-lowering drug other than ezetimibe on the initial day of therapy, and for patients who died during the period of the study. The follow-up period was between 4 and 31 months depending on the beginning of the therapy.

Data analyses were conducted according to the year during which therapy was started, the active ingredient and strength of the product(s) initially dispensed, and patient age and residence. Diagnostic data were limited to the International Classification of Diseases (ICD) code written on prescriptions, limiting the ability to determine whether therapy was aimed at primary or secondary prevention. On the basis of national therapeutic guidelines, however, it could be assumed that atorvastatin $80 \mathrm{mg}$ and ezetimibe were prescribed only for secondary prevention. 
In determining persistence, the first dispensing of a prescription was considered the start of study therapy. We considered therapy to have been discontinued if a patient did not obtain a replenishment of drug for at least 60 days (the "grace period"), according to the recommendation of the International Society of Pharmacoeconomics and Outcomes Research (ISPOR) [19]. An additional analysis was conducted for all patients treated with a statin using a grace period of 180 days. Data were examined and results reported in 1-month units; a month is the shortest time span for which the National Health Insurance Fund provides data. We attempted to determine the end of the therapy as accurately as possible by estimating the patient's actual use of an agent, assuming that, from the first prescription, a patient would have taken one tablet daily. Starting with the second prescription, we calculated how many days of therapy would have been possible with the number of tablets purchased to that date.

Study analyses were performed only for those patients whose consumption of tablets was calculated to be 0.75 to 1.25 times the number prescribed (calculated from the number of tablets dispensed and the time periods over which the tablets were dispensed). By doing so, small fluctuations could be excluded, which might be caused by interruption of therapy or patient stockpiling of drugs. In calculating general or total statin persistence, all statin products were considered together, disregarding switches to a different drug or a different dosage of the same drug.

For treatment groups other than the total statin group, we considered that initial therapy was terminated when a patient had a 60-day period during which the initial therapy was no longer taken exactly as prescribed, even if the patient then took a drug that was similar to the initial drug (e.g., atorvastatin or simvastatin-ezetimibe fixed combination instead of simvastatin), used the initial drug subsequently, or took a product with the same active ingredient as the initial drug but with a different dosage. For these specific groups, we therefore determined duration of persistence with the initially prescribed drug regimen, even though the prescription might have changed. The total statin group consisted of all patients who continued with any statin at any dose, while groups of users of individual statins consisted of only those who remained on the initially prescribed statin and dosage.

The National Health Insurance Fund database enabled us to study ezetimibe therapy, whether it consisted of ezetimibe monotherapy, ezetimibestatin fixed combination therapy, or therapy with ezetimibe plus a statin as separate products. We calculated the length of therapy from the first purchase of ezetimibe to the completion of the ezetimibe therapy, regardless of when the ezetimibe therapy was started. The start of ezetimibe therapy was not necessarily simultaneous with the start of statin therapy.

\section{Statistical analysis}

Survival analysis methods were used to assess persistence; censored data necessary for analyses were available. Survival curves were constructed according to Kaplan-Meier analysis (unadjusted); the ratio of patients still receiving therapy from month to month was calculated. Observations were censored when a person died or became institutionalized, or when the end of the study period was reached. We determined the medians of persistence and their 95\% Cls. The medians of persistence in different groups were compared with the Peto-Wilcoxon test.

\section{Results}

A total of 459034 patients started on any statin therapy: 188245 in 2007 and 209716 in 2008 (Table I). Therapy was initiated with atorvastatin in 277378 patients, simvastatin in 120 921, and rosuvastatin in 19687 . Therapy was initiated with ezetimibe in 8893 patients: ezetimibe monotherapy in 2112 , ezetimibe plus a statin in 2044, and a fixedcombination ezetimibe-statin product in 5145. Age groupings of patients ranged from $<20(n=2568)$ to $\geq 70$ years $(n=101301)$.

In the total group of patients who received statin therapy, 54\% discontinued during the first month, based on a grace period of 60 days. During the second month, $40.3 \%$ of patients were still receiving statin-based lipid-lowering treatment (Figure 1). This percentage decreased to $27 \%$ at 6 months and to $20.1 \%$ at the end of the first year (Table I). Because of the high degree of erosion (54\%) during the first month, an analysis was conducted based on a grace period of 180 days and yielded $27 \%$ persistence after 12 months. From the total 459034 patients in 5590 cases there were enough data about a former myocardial infarction or percutaneous coronary intervention in the database. In these cases the persistence at 1 month was $76 \%$, at 6 months $61 \%$, and at 12 months 50\%. These are favorable data, but not absolute. The real secondary prevention population might be higher.

During the first year of therapy, persistence for all statin therapy started in 2008 was slightly greater than for therapy started in 2007. During no month was the difference between years as much as $4 \%$. Therefore, although the difference between the median persistence of therapies started in 2007 and 2008 was statistically significant $(p<0.001)$, it was not deemed clinically relevant.

The median persistence for atorvastatin and simvastatin was 1 month, and for rosuvastatin it was 2 months (Table I); differences were statistically 
Table I. Groupings of patients by therapy, age, and geographic location, with numbers, median months of persistence, and persistence percentages at 6 and 12 months

\begin{tabular}{|c|c|c|c|}
\hline Group & $\begin{array}{l}\text { Number of } \\
\text { patients }\end{array}$ & $\begin{array}{l}\text { Persistence median in months } \\
\text { ( } 95 \% \text { confidence interval) }\end{array}$ & $\begin{array}{l}\text { Percentage of patients } \\
\text { persisting in therapy } \\
\text { at } 6 \text { and } 12 \text { months }\end{array}$ \\
\hline \multicolumn{4}{|l|}{ All (or total) statins* } \\
\hline All patients receiving statin therapy & 459034 & $1(1.1)$ & $27 \%, 20.1 \%$ \\
\hline 2007 start of statin therapy & 188245 & $1(1.1)$ & $25.4 \%, 20.3 \%$ \\
\hline 2008 start of statin therapy & 209716 & $1(1.1)$ & $27.4 \%, 19.9 \%$ \\
\hline \multicolumn{4}{|l|}{ Individual statin therapies** } \\
\hline Atorvastatin, all & 277378 & $1(1.1)$ & $27.3 \%, 20.9 \%$ \\
\hline Atorvastatin $10 \mathrm{mg}$ & 42037 & $1(1.1)$ & $22.7 \%, 16.1 \%$ \\
\hline Atorvastatin $20 \mathrm{mg}$ & 139850 & $1(1.1)$ & $24.5 \%, 17.5 \%$ \\
\hline Atorvastatin $40 \mathrm{mg}$ & 94678 & $1(1.1)$ & $20.2 \%, 14.5 \%$ \\
\hline Atorvastatin $80 \mathrm{mg}$ & 6409 & $1(1.1)$ & $13.2 \%, 8.2 \%$ \\
\hline Rosuvastatin, all & 19687 & $2(1.2)$ & $28.1 \%, 21.3 \%$ \\
\hline Simvastatin, all & 120921 & $1(1,1)$ & $26 \%, 20.5 \%$ \\
\hline Simvastatin $10 \mathrm{mg}$ & 12217 & $1(1,1)$ & $15.9 \%, 10 \%$ \\
\hline Simvastatin $20 \mathrm{mg}$ & 68778 & $1(1.1)$ & $21.6 \%, 15.3 \%$ \\
\hline Simvastatin $40 \mathrm{mg}$ & 41919 & $1(1.1)$ & $18.3 \%, 12.6 \%$ \\
\hline \multicolumn{4}{|l|}{ Age groups [years] ${ }^{\star \star \star}$} \\
\hline$<20$ & 2568 & $1(1.1)$ & $1 \%$, no data \\
\hline$\geq 20$ to $<30$ & 9084 & $1(1.1)$ & $6.9 \%, 3.7 \%$ \\
\hline$\geq 30$ to $<40$ & 30229 & $1(1.1)$ & $12.1 \%, 7.9 \%$ \\
\hline$\geq 40$ to $<50$ & 65137 & $1(1.1)$ & $17.6 \%, 11.8 \%$ \\
\hline$\geq 50$ to $<60$ & 139142 & $1(1.1)$ & $22.3 \%, 15.9 \%$ \\
\hline$\geq 60$ to $<70$ & 117096 & $1(1.1)$ & $28.6 \%, 21.5 \%$ \\
\hline$\geq 70$ & 101301 & $1(1.1)$ & $30.8 \%, 23.5 \%$ \\
\hline \multicolumn{4}{|l|}{ Ezetimibe ${ }^{\star \star \star \star}$} \\
\hline All ezetimibe groups & $n=8893$ & $2(2.2)$ & $36.6 \%, 26.7 \%$ \\
\hline Ezetimibemonotherapy & $n=2112$ & $1(1.1)$ & $24.1 \%, 16.6 \%$ \\
\hline Ezetimibe add-on to a statin & $n=2044$ & $3(3.4)$ & $39.4 \%, 25.5 \%$ \\
\hline Fixed ezetimibe-simvastatin combination & $n=5145$ & $3(3.3)$ & $38.5 \%, 27.5 \%$ \\
\hline
\end{tabular}

*This table contains patients with statin therapy only therefore patients with Ezetimibemonotherapy are not listed here. 61,073 patients started their therapy in 2009 Q1 period and their data was not compared to the patients started in 2007 or 2008 because of the much shorter follow up time. ${ }^{* *}$ Some initial therapies are not listed here, eg. fluvastatin, pravastatin because their importance is quite low in Hungary. In the active substance level (eg. simvastatin) the number of patients might be less than in the strength level (eg. simvastatin $10 \mathrm{mg}, 20 \mathrm{mg}, 40 \mathrm{mg}$ ). It is possible that a patient daily dosage is 1 pill during the initial simvastatin $10 \mathrm{mg}$ therapy so he/she is visible in the table. He/she may switch to simvastatin $20 \mathrm{mg}$ and during the therapy may have less or more than one pill dosage. In this case he/she is excluded from the examination in the active substance level. ***The National Health Insurance Found doesn't provide data for groups where the number of patients is less than 10. In the table of age groups 15 patients have been deleted for that reason because the original data was more detailed (age + agent). ${ }^{* * *} E z e t i m i b e ~ a d d-o n$ and fixed Ezetimibe therapy is possible for the same patient if he/she switches from add-on therapy to fixed combination therapy.

significant $(p<0.001)$. Persistence curves were also determined separately for the individual statins. The greatest difference between persistence curves was only $6 \%$ (observed in the first month), indicating that the differences were not of clinical significance.

Curves of persistence with different doses of simvastatin are shown in Figure 2. Persistence was greatest with the 20-mg dose, lower with the 40-mg dose, and lowest with the 10-mg dose. The difference between the groups was statistically significant $(p<0.001)$ but not clinically significant, with the greatest difference being only $6.3 \%$, seen in the third month.

Curves of persistence with different doses of atorvastatin are shown in Figure 3. After the first month, persistence decreased progressively in the following dose order: $20 \mathrm{mg}, 10 \mathrm{mg}, 40 \mathrm{mg}$, and 


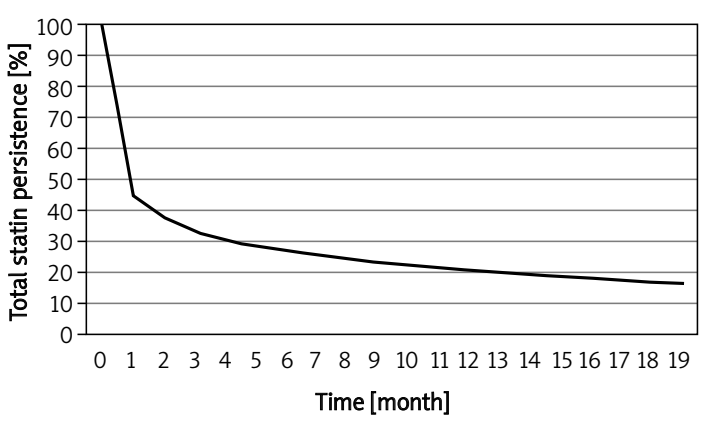

Figure 1. Kaplan-Meier plot of total statin persistence

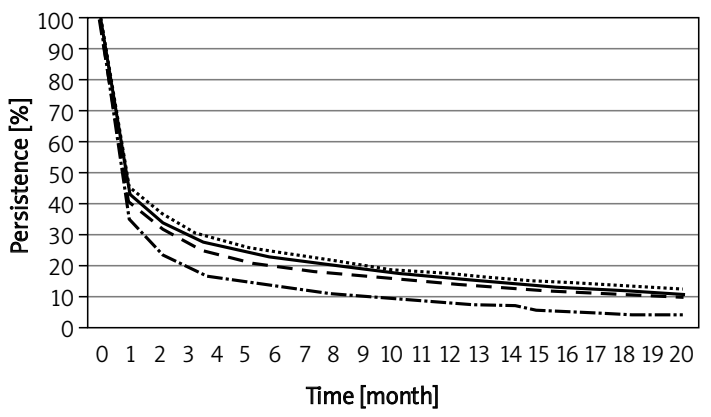

-Atorvastatin $10 \mathrm{mg} \quad$ …...Atorvastatin $20 \mathrm{mg}$

- -Atorvastatin $40 \mathrm{mg}$---Atorvastatin $80 \mathrm{mg}$

Figure 3. Kaplan-Meier plot of persistence for different atorvastatin doses

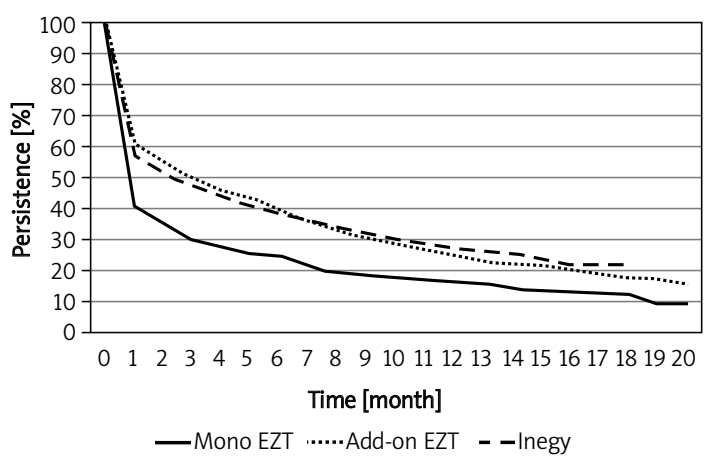

Figure 5. Kaplan-Meier plot of persistence for ezetimibe as monotherapy, add-on to a statin, and ezetimibe-statin fixed combination

finally $80 \mathrm{mg}$, which had a persistence considerably lower than that of the lower doses.

The persistence of initial therapies increased as the age of patients increased (Table I, Figure 4). At 6 months, persistence was $1 \%$ for the < 20-year age group and $30.8 \%$ for the $\geq 70$-year age group, an approximate $30 \%$ difference between the youngest and oldest age groups. The median persistence for all age groups was 1 month; the differences were statistically significant $(p<0.001)$.

Persistence data for different ezetimibe regimens are shown in Table I and Figure 5. The median per-

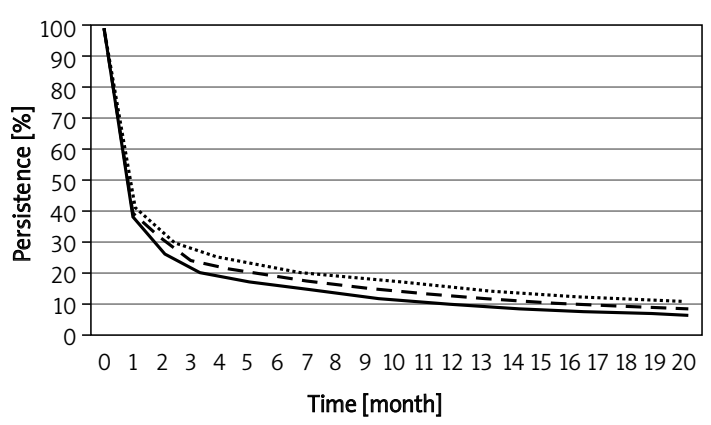

- Simvastatin $10 \mathrm{mg}$......Simvastatin $20 \mathrm{mg}$ - -Simvastatin $40 \mathrm{mg}$

Figure 2. Kaplan-Meier plot of persistence for different simvastatin doses

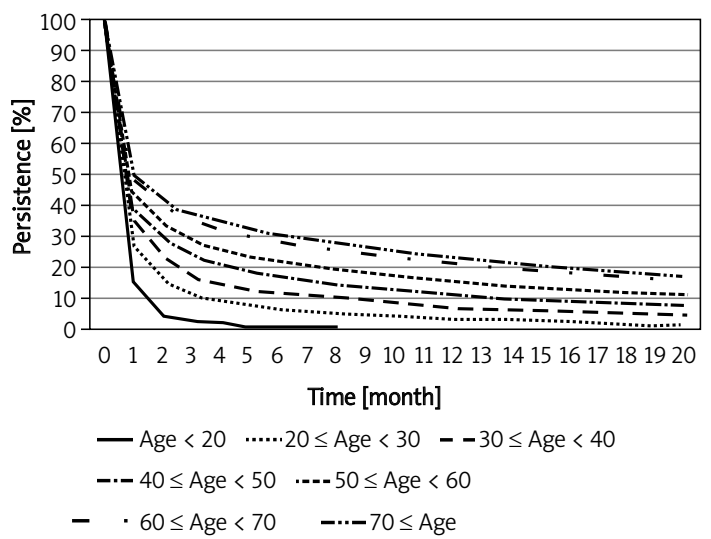

Figure 4. Kaplan-Meier plot of persistence for different age cohorts

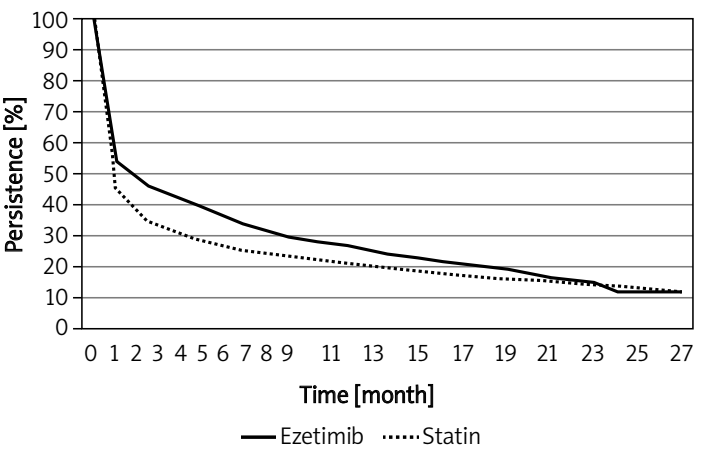

Figure 6. Kaplan-Meier plot of total ezetimibe and total statin persistence

sistence with ezetimibe monotherapy was 1 month and that for ezetimibe added to statin or for fixed ezetimibe-statin combination therapy was 3 months. There was no statistically significant difference between the add-on and fixed combination regimens $(p=0.94)$. Persistence with all ezetimibe therapies (median 2 months) was greater than for simvastatin and atorvastatin therapies (Table I and Figure 6).

\section{Discussion}

This is the first study, to our knowledge, of detailed persistence patterns with statin therapy 
among Hungarian patients. We determined that when persistence was based on a 60-day grace period, the percentage of patients who persisted with therapy was only $46 \%$ after 1 month, $40.3 \%$ after 2 months, $27 \%$ after 6 months, and $20.1 \%$ after 12 months. Almost $80 \%$ of patients had discontinued therapy during the first year. When persistence was based on a more lenient grace period of 180 days, persistence after 12 months was increased only from $20.1 \%$ to $27 \%$. These results indicate that, because of early discontinuation, a considerable proportion of Hungarian patients do not experience advantages of statin therapy. Several studies have shown that the duration of statin therapy can be a major factor in determining therapeutic benefit; therapy that is not sufficiently persistent may provide no significant cardioprotection $[14,15]$.

Contrary to our expectation, persistence with statin therapy in Hungary was not as great as in developed countries. Compared with the 1-month median persistence we found, Kamal-Bahl et al., using less strict criteria than the current study for determining discontinuation, found a median persistence of 27.5 months in the United States (US) [27]. In another US study, Foody et al. used discontinuation criteria similar to this study, and found 12-month persistence for atorvastatin in primary prevention to be about $40 \%$, nearly twice as high as our finding of $20.9 \%$; for simvastatin, they found 12-month persistence to be about 30\%, compared to $20.5 \%$ for our patients [28]. Larsen et al., using a 30 -day grace period, found that only $11.2 \%$ of Danish patients dropped out of therapy after 1 month [29], compared with $54 \%$ of Hungarian patients. The Mantel-Teeuwisse et al. study in the Netherlands showed significantly better 1-year persistence than in our study: $61.5 \%$ vs. $20.1 \%$ [30]. Persistence rates higher than those in our study were also observed by Perreault et al. in Quebec [31], HelinSalmivaara et al. in Finland [32], Deambrosis et al. in Italy [33], and Chodick et al. in Israel [34].

However, our study is not the only one to have found a disappointingly rapid drop in drug usage after initiation of therapy for primary or secondary prevention of chronic conditions. In several large, retrospective cohort studies of statin use in the US and Canada, the greatest decline in persistence occurred during the first 3-6 months of treatment, with $25 \%$ to almost $50 \%$ of patients discontinuing statins within 6 months of starting therapy [35]. In a North American cohort study of elderly patients (65 years or older), almost $15 \%$ of the cohort discontinued lipid-lowering therapy after their initial prescription fill, and more than one-third of patients discontinued treatment within 1 year [36]. The study by Chodick et al. in Israel found that nearly onethird of the primary-prevention cohort and onequarter of the secondary-prevention cohort dis- continued statin treatment after only one prescription fill [34]. These observations are consistent with the health belief model, according to which the likelihood of adherence to a medication regimen is determined by the perceived threat of disease (susceptibility and severity) and perceived benefits of therapy [37]. Given that hyperlipidemia is an asymptomatic disease until a cardiovascular event occurs, the perceived threat of disease and benefits of lipid-lowering therapy may not be tangible to many patients, compromising adherence and persistence.

Our study included fairly adherent patients, by restricting data analyses to patients who were taking 0.75-1.25 times the number of tablets prescribed for them. We could not determine whether patients who took twice as long as they should have to obtain a renewal prescription were cutting their tablets in half and were regularly taking half their prescribed dose daily or were taking a full tablet every other day. Results were affected to a non-significant extent (by $8 \%$ to $17 \%$ ) by eliminating from the analysis the data from those patients who refilled prescriptions regularly but less frequently $(<0.75)$ or more frequently $(>1.25)$ than called for by their prescription.

Medication adherence and persistence are interrelated, and both impact clinical outcomes of treatment [12]. Wisniowska and Skowron in Poland found persistence with statin therapy to be 160 days for highly adherent $(\geq 80 \%)$ patients and 90 days for poorly adherent $(<80 \%)$ patients [38]. The difference in our study between 1-year persistence based on 60 - and 180 -day grace periods indicates that fewer than $7 \%$ of patients who were non-adherent for a 60-day grace period resumed therapy during the subsequent 120 days.

A recent systematic review of 19 studies examining the relationship between adherence or persistence to statin therapy and clinical outcomes indicated that high levels of adherence and longer durations of persistence with statins are associated with progressively increasing clinical benefits in primary and secondary prevention populations, including significant reductions in all-cause mortality (OR 0.49-0.66), fatal and nonfatal coronary heart disease events (OR 0.74-0.83), and hospitalizations (OR 0.19-0.70) [15]. A recent comparative cost-effectiveness analysis determined that both reminders/educational materials and pharmacist/nurse management programs were cost-effective interventions to improve adherence with CVD medications associated with incremental cost-effectiveness ratios (ICER) of \$4984 and \$6358 per quality-adjusted life-year (QALY) gained, respectively [39]. According to a World Health Organization report on medication adherence to long-term therapies, "Increasing the effectiveness of adherence 
interventions may have a far greater impact on the health of the population than any improvement in specific medical treatments" [40].

The increased persistence observed in our study when comparing therapy begun in 2008 with that begun in 2007 was not markedly improved, but may signal a trend toward continuing improvement over time.

We found that discontinuation of therapy decreased with increasing age; older patients tended to be more adherent to physicians' instructions. In the sixth month of therapy, there was approximately a $30 \%$ difference in median persistence between the youngest and oldest age groups. Although this difference was statistically significant ( $p<0.001$ ), the median persistence for all groups was only 1 month. One month was also the median persistence for the total statin group and the groups of users of individual statins (other than rosuvastatin, which had a median persistence of 2 months). Thus, the percent of patients dropping out of therapy early is considerable for all age and therapy groups, regardless of any statin switching or dose changing that may have taken place. The causes of the discontinuation were not studied. On reason might be that the statin induced muscle complaints, the frequency of which in observation studies could be $5-10 \%$ [41]. In a prospective cohort study investigating the effect of physical activity and statins conducted on more than 10000 patients during a 10-year follow-up the mortality risk was significantly better in people taking statins than in those not taking statins (18.5\% vs. $27.7 \%)$ $(p<0.0001)$. In patients who took statins, mortality risk decreased as fitness increased (a similar trend between fitness and mortality was observed in patients without statins) [42].

Other studies have yielded conflicting results regarding association of persistence with age. Several found, as have we, that persistence improves with increasing patient age (up to a certain 'threshold' in some cases [e.g., 55-64 years of age], beyond which persistence declines) [30, 43, 44], while others found older age to be a predictor of poor longterm persistence [28, 36].

The median persistence with combined ezetimibe and statin therapy - whether ezetimibe was added on to a statin or was prescribed as an ezetimibe-statin fixed combination - was 3 months, which was longer than the 1-month persistence with total statin therapy. Greater persistence with combination ezetimibe and statin therapy than with total statin therapy was observed through a full year: $25.5 \%$ with ezetimibe add-on therapy and $27.5 \%$ with fixed-combination therapy compared to $20.1 \%$ at 12 months for total statin persistence.

Based on the Hungarian subsidy system, we were able to consider treatments containing eze- timibe prescribed for patients with CVD as secondary prevention treatments. We could not determine whether statin monotherapy was used for primary or secondary prevention, other than for atorvastatin 80-mg therapy, which, in Hungary, is subsidized only for secondary prevention. Several groups of investigators have reported that patients receiving secondary-prevention therapy were more adherent to their drug regimen than those receiving primary-prevention therapy, possibly due to an increased appreciation of the importance of managing their risk factors) [33, 43, 44].

Kamal-Bahl et al. studied a US population with private health insurance and found that, using a 180-day grace period, persistence after 1 year was greater for statin users $(71.1 \%)$ than for ezetimibe users (67\%) [27]. These investigators did not specify whether therapy was used for primary or secondary prevention.

There is a limitation of this investigation in that we studied only those prescriptions that were subsidized by the National Health Insurance Fund. Undoubtedly there were patients who were persistent with statin therapy but were unknown to us because they were able to continue taking a drug for longer than the 60-day grace period without applying for a fund subsidy. These patients may have received the drug while in the hospital, been discharged from the hospital with a supply of the drug, used drugs left over from a relative's or friend's supply, or received samples of drugs.

We did not assess proportions of patients making and keeping appointments for follow-up lipid tests and physician visits, factors which can impact adherence to therapy. In a large cohort study, Benner et al. demonstrated that patients who received follow-up physician visits and lipid tests were $45 \%$ more likely to be adherent $(95 \% \mathrm{Cl} 1.34-1.55)$ to lipidlowering therapy [45].

In conclusion, we can state that the low rate of persistence with statin therapy that we observed in Hungary is likely associated with occurrence of cardiovascular events or deaths that otherwise might have been prevented by appropriately longterm continuation of statin therapy. These data are consistent with a likely financial drain on national resources used to subsidize suboptimal statin therapy, with insufficient yields of health improvement in the Hungarian population. Persistence with lipidlowering therapy among Hungarian patients needs to be increased. The key to improvement is the patient-physician relation. Every time a patient meets a health care professional the attention for the importance of taking the pills properly has to be drawn up. The possible side effects must be discussed in detail. The patients' motivation could be increased by explaining the role of cholesterol in the prevention of atherosclerosis. Broad professional 
cooperative efforts, including those of pharmacists, in order to optimize the beneficial effects of lipid therapies, to improve the patients' persistence, could further reduce cardiovascular morbidity and mortality.

\section{Acknowledgments}

Data from the Hungarian Institutional Database of the National Health Insurance Fund were used in the study.

\section{References}

1. Józan P. Changes in the socio-economic system and political regime and the fourth stage of the epidemiological transition [Hungarian]. Orv Hetil 2012; 153: 662-77.

2. Helis E, Augustincic L, Steiner S, et al. Time trends in cardiovascular and all-cause mortality in the 'old' and 'new' European Union countries. Eur J Cardiovasc Prev Rehabil 2011; 18: 347-59.

3. Szucs TD. Healing unhealthy Hungarian hearts - at what cost? With what benefit? Cardiovasc Drugs Ther 2006; 20: 239-43.

4. Mercando AD, Lai HM, Aronow WS, et al. Reduction in atherosclerotic events: a retrospective study in an outpatient cardiology practice. Arch Med Sci 2012; 8: 57-62.

5. Lai HM, Aronow WS, Mercando AD, et al. The impact of statin therapy on long-term cardiovascular outcomes in an outpatient cardiology practice. Arch Med Sci 2012; 8: 53-56.

6. Mark L, Paragh GY, Karadi I, et al. Changes in attainment of lipid goals by general practitioners and specialists in patients at high cardiovascular risk in Hungary during 2004-2008. Arch Med Sci 2010; 6: 695-700.

7. Mark L, Paragh GY, Karadi I, et al. An attempt to make lipid-lowering therapy more effective in Hungary. The results of MULTI GAP 2010 and the Plus Program. Arch Med Sci 2011; 7: 760-6.

8. Mark L, Paragh GY, Karadi I, et al. How can we further improve the LDL-cholesterol target level achievement rate based on the Hungarian MULTI GAP 2011 study results and considering the new European dyslipidaemia guidelines? Arch Med Sci 2012; 8: 608-13.

9. Ray KK, Cannon CP, Braunwald E. Recent trials of lipid lowering. Int J Clin Pract 2007; 61: 1145-59.

10. Cholesterol Treatment Trialists' (CTT) Collaboration. Efficacy and safety of more intensive lowering of LDL cholesterol: a meta-analysis of data from 170000 participants in 26 randomised trials. Lancet 2010; 376: 1670-81.

11. Peterson AM, Nau DP, Cramer JA, et al. A checklist for medication compliance and persistence studies using retrospective databases. Value Health 2007; 10: 3-12.

12. Cramer JA, Roy A, Burrell A, et al. Medication compliance and persistence: terminology and definitions. Value Health 2008; 11: 44-7.

13. Cardinal H, Monfared AA, Dorais M, et al. The concept of the 'percent wasted patients' in preventive health strategies. Pharmacoepidemiol Drug Saf 2006; 15: 57-61.

14. Downs JR, Clearfield $M$, Weis $S$, et al. Primary prevention of acute coronary events with lovastatin in men and women with average cholesterol levels: results of AFCAPS/ TexCAPS. Air Force/Texas Coronary Atherosclerosis Prevention Study. JAMA 1998; 279: 1615-22.

15. Simpson RJ Jr, Mendys P. The effects of adherence and persistence on clinical outcomes in patients treated with statins: a systematic review. J Clin Lipidol 2010; 4: 462-71.
16. Ho PM, Spertus JA, Masoudi FA, et al. Impact of medication therapy discontinuation on mortality after myocardial infarction. Arch Intern Med 2006; 166: 1842-7.

17. Moride Y, Hegele RA, Langer A, et al. Clinical and public health assessment of benefits and risks of statins in primary prevention of coronary events: resolved and unresolved issues. Can J Cardiol 2008; 24: 293-300.

18. Perreault S, Blais L, Lamarre D, et al. Persistence and determinants of statin therapy among middle-aged patients for primary and secondary prevention. $\mathrm{Br} J$ Clin Pharmacol 2005; 59: 564-73.

19. Osterberg L, Blaschke T. Adherence to medication. N Engl J Med 2005; 353: 487-97.

20. McHorney CA. The adherence estimator: a brief, proximal screener for patient propensity to adhere to prescription medications for chronic disease. Curr Med Res Opin 2009; 25: 215-38.

21. Poluzzi E, Piccinni C, Carta P, et al. Cardiovascular events in statin recipients: impact of adherence to treatment in a 3-year record linkage study. Eur J Clin Pharmacol 2011; 67: 407-14.

22. Rasmussen JN, Chong A, Alter DA. Relationship between adherence to evidence-based pharmacotherapy and longterm mortality after acute myocardial infarction. JAMA 2007; 297: 177-86.

23. Lindgren $\mathrm{P}$, Eriksson J, Buxton $\mathrm{M}$, et al.; the ASCOT trial investigators. The economic consequences of nonadherence to lipid-lowering therapy: results from the Anglo-Scandinavian-Cardiac Outcomes Trial. Int J Clin Pract 2010; 64: 1228-34.

24. Aubert RE, Yao J, Xia F, Garavaglia SB. Is there a relationship between early statin compliance and a reduction in healthcare utilization? Am J Manag Care 2010; 16: 459-66.

25. Gadkari AS, McHorney CA. Medication nonfulfillment rates and reasons: narrative systematic review. Curr Med Res Opin 2010; 26: 683-705.

26. Jermendy G, Wittmann I, Nagy L, Kiss Z, et al. Persistence of initial oral antidiabetic treatment in patients with type 2 diabetes mellitus. Med Sci Monit 2012; 18: CR72-7.

27. Kamal-Bahl SJ, Burke T, Watson D, et al. Discontinuation of lipid modifying drugs among commercially insured United States patients in recent clinical practice. Am J Cardiol 2007; 99: 530-4.

28. Foody JM, Joyce AT, Rudolph AE, et al. Persistence of atorvastatin and simvastatin among patients with and without prior cardiovascular diseases: a US managed care study. Curr Med Res Opin 2008; 24: 1987-2000.

29. Larsen J, Andersen M, Kragstrup J, et al. High persistence of statin use in a Danish population: compliance study 1993-1998. Br J Clin Pharmacol 2002; 53: 375-8.

30. Mantel-Teeuwisse AK, Goettsch WG, Klungel OH, et al. Long term persistence with statin treatment in daily medical practice. Heart 2004; 90: 1065-6.

31. Perreault S, Blais L, Dragomir A, et al. Persistence and determinants of statin therapy among middle-aged patients free of cardiovascular disease. Eur J Clin Pharmacol 2005; 61: 667-74.

32. Helin-Salmivaara A, Lavikainen P, Ruokoniemi P, et al. Persistence with statin therapy in diabetic and nondiabetic persons: a nation-wide register study in 19952005 in Finland. Diabetes Res Clin Pract 2009; 84: e9-11

33. Deambrosis P, Saramin C, Terrazzani G, et al. Evaluation of the prescription and utilization patterns of statins in an Italian local health unit during the period 1994-2003. Eur J Clin Pharmacol 2007; 63: 197-203.

34. Chodick G, Shalev V, Gerber Y, et al. Long-term persistence with statin treatment in a not-for-profit health main- 
tenance organization: a population-based retrospective cohort study in Israel. Clin Ther 2008; 30: 2167-79.

35. Jackevicius CA, Mamdani M, Tu JV. Adherence with statin therapy in elderly patients with and without acute coronary syndromes. JAMA 2002; 288: 462-7.

36. Benner JS, Glynn RJ, Mogun H, et al. Long-term persistence in use of statin therapy in elderly patients. JAMA 2002; 288: 455-61.

37. Rosenstock IM, Strecher VJ, Becker MH. Social learning theory and the Health Belief Model. Health Educ Q 1988; 15: $175-83$.

38. Wisniowska B, Skowron A. Evaluation of patients' adherence to statins in Poland. Curr Med Res Opin 2011; 27: 99-105.

39. Chapman RH, Kowal SL, Cherry SB, et al. The modeled lifetime cost-effectiveness of published adherenceimproving interventions for antihypertensive and lipidlowering medications. Value Health 2010; 13: 685-94.

40. World Health Organization. Adherence to long-term therapies. Evidence for action. World Health Organization 2003. http://www.who.int/chp/knowledge/publications/ adherence_report/en/. Accessed January 7, 2011.

41. Bruckert E, Hayem G, Dejager S, et al. Mild to moderate muscular symptoms with high-dosage statin therapy in hyperlipidemic patients: the PRIMO study. Cardiovasc Drugs Ther 2005; 19: 403-14.

42. Kokkinos PF, Faselis C, Myers J, et al. Interactive effects of fitness and statin treatment on mortality risk in veterans with dyslipidaemia: a cohort study. Lancet 2013; 381: 394-9.

43. Benner JS, Chapman RH, Petrilla AA, Tank SS, Rosenberg N, Schwartz JS. Association between prescription burden and medication adherence in patients initiating antihypertensive and lipid-lowering therapy. Am J Health Syst Pharm 2009; 66: 1471-7.

44. Chapman RH, Benner JS, Petrilla AA, et al. Predictors of adherence with antihypertensive and lipid-lowering therapy. Arch Intern Med 2005; 165: 1147-52.

45. Benner JS, Tierce JC, Ballantyne CM, et al. Follow-up lipid tests and physician visits are associated with improved adherence to statin therapy. Pharmacoeconomics 2004; 22 (suppl. 3): 13-23. 